\title{
Susceptibilities and screening masses in two flavor QCD
}

\author{
Rajiv V. Gavai, , Sourendu Gupta, 周 and Pushan Majumdar \\ Department of Theoretical Physics, \\ Tata Institute of Fundamental Research, \\ Homi Bhabha Road, Mumbai 400005, India.
}

\begin{abstract}
We studied QCD with two flavors of dynamical staggered quarks at finite temperature, with a bare sea quark mass of about $17 \mathrm{MeV}$. We report investigations of baryon, isospin, charge and strangeness susceptibilities, as well as screening masses obtained from correlators of local and onelink separated meson operators. These were studied as functions of valence quark mass at several temperatures. Our results for susceptibilities deviate significantly from ideal gas values, and even more from the weak coupling series. We also report the first measurement of off-diagonal quark number susceptibilities below the transition temperature, $T_{c}$, where they are the main contribution to charge fluctuations. We present evidence for a close connection between the susceptibilities and the screening masses.
\end{abstract}

PACS numbers: 11.15.Ha, 12.38.Mh

\section{INTRODUCTION}

Experiments at the Brookhaven RHIC are now seeking to establish the formation of quark-gluon plasma. Proposals for experimental signatures of the plasma have to take into account its physical nature. Such information is most reliably obtained in lattice simulations of the hot phase of QCD. At present the phase transition temperature, $T_{c}$, is fairly well known [1, 2], and reasonably reliable information on the equation of state has also been obtained [3]. Screening and fluctuations of conserved charges are some of the other relevant properties of the plasma. We report extensive new lattice results for these.

We address two different physics questions in this paper. We are primarily interested in the question of baryon number and electric charge fluctuations in the plasma. These are quantities of direct experimental relevance, as has been realized recently [4, 河. We construct them from measurements of quark number susceptibilities [6]. In addition, we study screening masses in the plasma through the study of spatial correlators, paying special attention to their tensor structures. This gives a new relation between the susceptibilities and screening masses which are verified by our lattice measurements. In particular, we explain why non-perturbative phenomena in the latter are closely connected with deviations from perturbation theory in the former.

Temperatures just above $T_{c}$ are likely to be the most relevant region for applications in heavy ion physics and cosmology. Lattice measurements of the equation of state deviate significantly from the usual high temperature perturbative expansion. This stimulated many attempts to resum the weak coupling expansion, with varying degrees of success [7]. Many of these techniques would have definite predictions for the susceptibilities that we measure. Since our measurements also deviate from the weak coupling expansion, they stand as yet another invitation to resum the weak coupling series.

While the world contains six flavors of quarks, it suffices to consider two light ( $u$ and d) and a moderately heavy (s) quark for the physics of the QCD phase transition. Starting from a quenched theory where all quark loop effects are turned off, one can envisage successive approximations by which dynamical quarks are switched on in the sequence of their masses. Such an incremental strategy is actually forced by the cost of lattice simulations of full QCD. Earlier we simulated QCD in the quenched approximation, and reported our measurements of various quark susceptibilities [8]. In the simulations we report here, we go to the next level of approximation by using dynamical $\mathrm{u}$ and $\mathrm{d}$ quarks with a bare mass of about $17 \mathrm{MeV}$. Our results indicate that at temperatures above $T_{c}$ the change due to the inclusion of light quarks is small. Unquenching the heavier strange quark may thus be correspondingly less important. Even in the cold phase below $T_{c}$, taking still lighter $\mathrm{u}$ and $\mathrm{d}$ quarks may be more crucial in future than including dynamical strange quarks.

The plan of this paper is the following - technical material on the simulations, including the lattice scales and

\footnotetext{
*Electronic address: gavai@tifr.res.in

${ }^{\dagger}$ Electronic address:

${ }^{\ddagger}$ Electronic address: pushan@theory.tifr.res.in
} 
details of the data taking procedure, is presented in the next section. The definitions and notation, and details of measurements of the screening masses and quark number susceptibilities are given in the following two sections, in that order. The discussion and summary in the final section is designed to facilitate use by those who are mainly interested in applications of our results or wish to have an overview of future directions in lattice measurements of susceptibilities.

\section{THE SIMULATIONS AND SCALES}

\section{A. Run parameters}

\begin{tabular}{|c|c|c|c|c|}
\hline$T / T_{c}$ & $m a$ & $\beta$ & size & statistics \\
\hline 0.00 & 0.025 & 5.2875 & $12^{4}$ & 81 \\
1.00 & 0.025 & 5.2875 & $4 \times 12^{3}$ & 249 \\
& & & $4 \times 16^{3}$ & 42 \\
1.25 & 0.02 & 5.35 & $4 \times 12^{3}$ & 50 \\
1.50 & 0.0125 & 5.420 & $4 \times 12^{3}$ & 50 \\
1.50 & 0.0167 & 5.429 & $4 \times 8^{3}$ & 50 \\
& & & $4 \times 12^{3}$ & 50 \\
& & & $4 \times 16^{3}$ & 20 \\
& & & $4 \times 16 \times 10^{2}$ & 2001 \\
& & & $4 \times 16 \times 10^{2}$ & $124^{*}$ \\
& & & $4 \times 24 \times 10^{2}$ & 1500 \\
2.00 & 0.0125 & 5.540 & $4 \times 12^{3}$ & 50 \\
& & & $4 \times 16 \times 10^{2}$ & 2021 \\
3.00 & 0.0083 & 5.675 & $4 \times 12^{3}$ & 50 \\
& & & $4 \times 16^{3}$ & 1139 \\
\hline
\end{tabular}

TABLE I: Details of $N_{f}=2$ runs. The runs were made with a trajectory length of 1 MD time unit, a time step of 0.01 and a conjugate gradient stopping criterion of $10^{-5} \sqrt{V}$. The starred run was performed with half the MD time step but the same trajectory length.

\begin{tabular}{|c|c|c|c|c|}
\hline$T / T_{c}$ & $m a$ & $\beta$ & size & statistics \\
\hline 1.5 & 0.0167 & 5.8941 & $4 \times 24 \times 10^{2}$ & 800 \\
2.0 & 0.0125 & 6.0625 & $4 \times 16 \times 10^{2}$ & 6500 \\
& & & $4 \times 24 \times 10^{2}$ & 850 \\
3.0 & 0.0083 & 6.3375 & $4 \times 16 \times 14^{2}$ & 7500 \\
\hline
\end{tabular}

TABLE II: Details of new quenched runs. These runs supplement earlier ones discussed in [8].

We have simulated QCD with two flavors of light dynamical staggered quarks $\left(N_{f}=2\right)$ with the R-algorithm [9] on $N_{t} \times N_{x}^{2} \times N_{z}$ lattices with $N_{t}=4$. For investigations of quark number susceptibilities, we chose spatially symmetric lattices with $N_{x}=N_{z}$. On the other hand, screening correlators were easier to measure on elongated lattices with $N_{z}>N_{x}$. Most of our simulations consisted of generating dynamical configurations with two flavors $\left(N_{f}=2\right)$ of dynamical staggered quarks with the sea quark mass, $m$, held fixed in physical units at $m / T_{c}=0.1$ as the temperature was varied. A small set of runs with $m / T_{c}=0.075$ was performed to check the magnitude of sea quark mass effects.

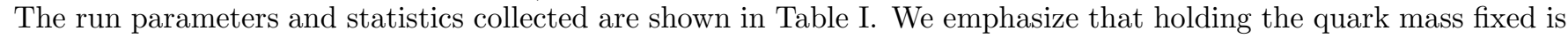
important for physics applications, whereas the older measurements fixed $m / T$ instead.

After a few tuning runs which reproduced the results of previous studies [9, 10], we chose to run with parameters similar to those used in previous works with two flavors of staggered quarks, i.e., trajectory length of 1 molecular dynamics (MD) time unit, integrated in 100 steps of $0.01 \mathrm{MD}$ time units each, and a conjugate gradient stopping criterion of $10^{-5} \sqrt{V}$ on the modulus of the remainder vector $\left(V=N_{x}^{2} N_{z} N_{t}\right)$. It should be noted that very similar run 
parameters are often used in $N_{f}=4$ Hybrid Monte Carlo (HMC) runs, where a Metropolis choice ensures that the correct weight for a configuration is obtained even for a finite number of time steps. For $N_{f}=2$ a bias-free estimate of the weight is not guaranteed. In order to estimate systematic errors from this source we also made a test run with half the step size but for the same trajectory length.

Part of the purpose of this work is a systematic comparison of screening masses with different numbers of dynamical quarks. Details of our $N_{f}=4$ simulations are available elsewhere [11, 12]. Similar details of runs in quenched QCD have been presented in [8]. However, we also generated a few sets of new quenched configurations. Details of the algorithm and data taking remain as in [8]. The run parameters and statistics for these quenched runs are listed in Table II.

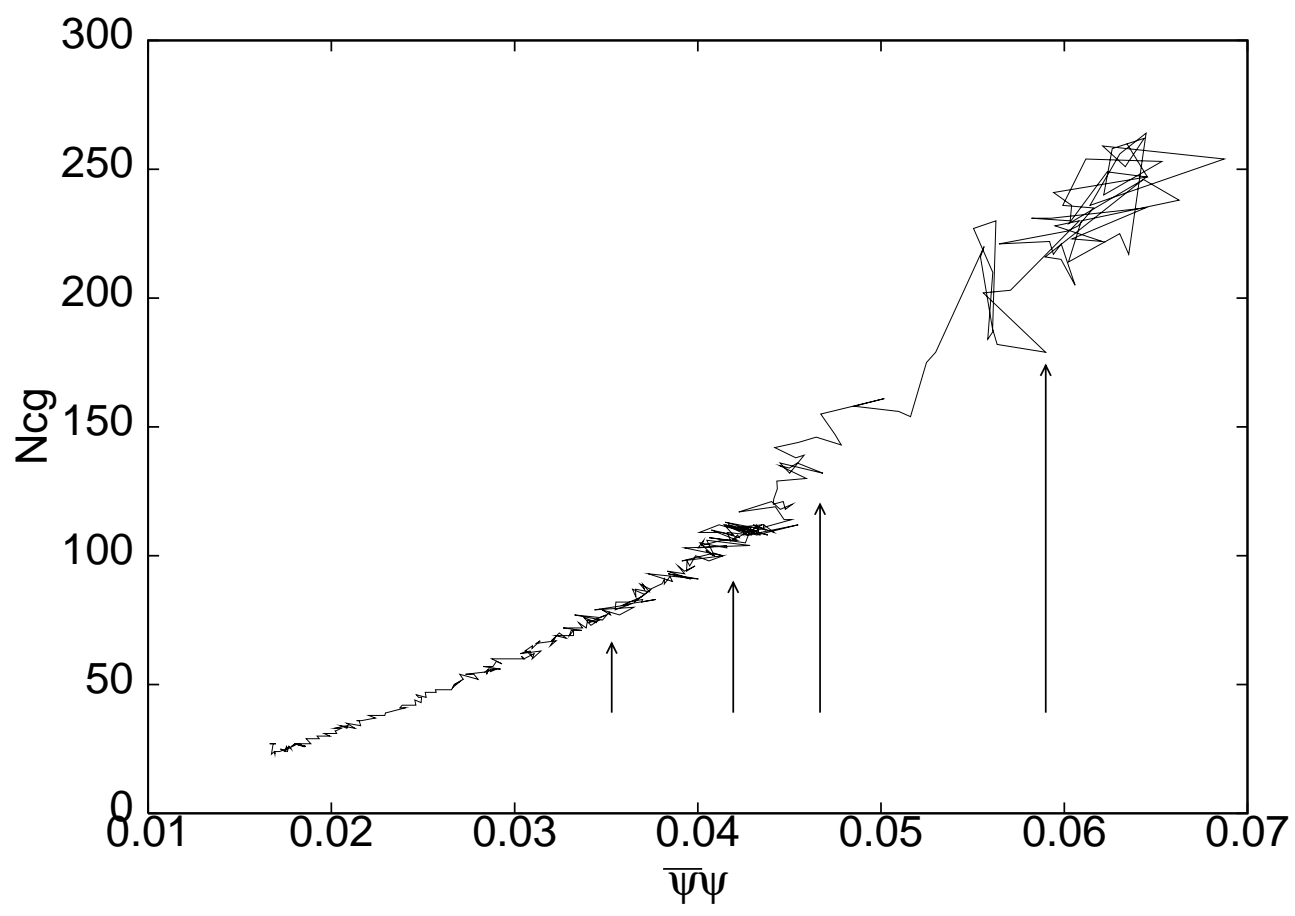

FIG. 1: A diagnostic for thermalisation. The points at which the trajectory length was changed are marked by vertical arrows.

\section{B. Setting the scale}

Relative temperature scales, i.e., $T / T_{c}$, can be deduced from previous estimates of critical couplings for various values of $N_{t}$ at the quark masses of interest to us [10]. Thus, the temperature of a run with $N_{t}=4$ at $\beta_{c}\left(N_{t}^{\prime}\right)$ is $T / T_{c}=N_{t}^{\prime} / 4$. Since $T_{c}$ is known to be $167 \pm 17 \mathrm{MeV}$ in physical units [2], this allows us to deduce the value of lattice spacing, $a$, in physical units using the relation $T=1 /\left(N_{t} a\right)$. For those values of $T$ which cannot be obtained by simulations on lattices with different $N_{t}$ we use the two-loop beta-function of QCD to deduce the lattice spacing, and hence the temperature. A recent global analysis of data assures us that this is possible [2]. The values of the inverse lattice spacings appropriate to our measurements are given in Table II. The sea quark masses that we use are $m=17 \pm 2 \mathrm{MeV}\left(m / T_{c}=0.1\right)$ and $12 \pm 1 \mathrm{MeV}\left(m / T_{c}=0.075\right)$.

Another way of setting the lattice spacing is to use previous lattice measurements of the $\rho$ meson mass with the same quark mass and coupling [13]. This gives us a lattice spacing $1 / a=580 \pm 20 \mathrm{MeV}$ at $T_{c}$, and hence the dynamical sea quark mass comes out to be $m=14.5 \pm 0.6 \mathrm{MeV}\left(m / T_{c}=0.1\right)$ and $m=10.9 \pm 0.4\left(m / T_{c}=0.075\right)$. This consistency between the two estimates is, of course, not unexpected, since it is well known that $T_{c} / m_{\rho}$ is reasonably independent of lattice spacing 10 .

The strange quark mass can be chosen in two different ways. One is to take the strange quark mass to be 75 $170 \mathrm{MeV}$ [14]. This gives $m_{s} / T_{c}=0.41-1.0$ [22]. Alternatively, we can use the ratio $m_{s} / m_{d}=17-25$ [14 to get $m_{s} / T_{c}=1.7-2.5$. Clearly, in the limit when the light quark masses are more realistic, the two procedures should give similar values. However, the heavier the strange quark mass is, the more strongly will it be subject to lattice artifacts. In view of this, we have chosen to work with $m_{s} / T_{c}=0.75$, corresponding to $m_{s}=125 \mathrm{MeV}$. A realistic ratio of the 
strange and light quark masses will thus need future simulations with lighter $\mathrm{u}$ and d quark masses. Furthermore, the fact that the strange quark is reasonably heavy in lattice units indicates that the unquenching effects on it would be small, at least near $T_{c}$.

\section{Thermalisation and autocorrelations}

Due to the fact that each configuration takes a long time to generate, a major issue in any Fermion simulation is to identify when a run has thermalised. We monitored thermalisation through measurements of plaquettes, Wilson line, the chiral condensate and the number of conjugate gradient iterations needed. Since plaquette measurements are not very noisy, these were a good estimator of the approach to equilibrium. At temperatures above $T_{c}$, the difference of purely spatial and mixed time-space plaquettes is nonzero, and it turns out to develop late during equilibration. This was one of the best criteria we observed for closeness of approach to equilibrium. However, even more efficient was a scatter plot of the stochastic estimator of the condensate $(\bar{\psi} \psi)$ against the number of conjugate gradient iterations $\left(N_{c g}\right)$. As shown in Figure $\mathbb{1}$, a very definite directional movement occurs away from equilibrium, but as equilibrium is approached the movement becomes a random walk within a well defined area in the plane of $\bar{\psi} \psi$ and $N_{c g}$. Monitoring this scatter plot thus offered a possibility of tuning the algorithm to achieve fast thermalisation.

We did this by starting each run from an ordered configuration with small trajectory lengths $(t \simeq 0.05 \mathrm{MD}$ units). As the simulation proceeded, the plot of $\bar{\psi} \psi$ against $N_{c g}$ began to slow. When this happened we increased the trajectory length in small steps until it reached unity or resulted in further motion. A post-facto check of the tuning was obtained after subsequent runs verified that the system continued to perform a random walk without directed movement. This method was developed in our earlier $N_{f}=4 \mathrm{HMC}$ runs and worked well also for these $N_{f}=2 \mathrm{HMD}$ runs. Using this technique we often managed to achieve thermalisation in 20-30 units of MD time.

A second issue in the control of computer time is the matter of how often measurements of an observable can be made so that two successive measurements are effectively decorrelated. We have estimated autocorrelation times from a variety of gauge and fermion observables and found them to be small. Local operators such as plaquettes seem to be essentially decorrelated in about 2 trajectories. Even long distance observables, such as the pion screening correlator at the longest possible distance, are decorrelated in about 2-3 trajectories. In view of these results, we have taken data for screening masses on every second trajectory, and for susceptibilities every tenth trajectory. This is justified, a posteriori, by the fact that none of the screening masses is found to be very small.

\section{SCREENING CORRELATORS AND MASSES}

\section{A. Symmetries and transfer matrices}

Screening involves the transfer matrix in a spatial direction of the Euclidean finite temperature lattice. Thus, screening correlators and masses are classified according to the symmetries of this transfer matrix, Screening masses are obtained from the exponential decay of the screening correlators

$$
C(z)=\left\langle\frac{1}{N_{x}^{2} N_{t}} \sum_{x, y, t} M(0) M^{\dagger}(x, y, z, t)\right\rangle .
$$

The operators $M(x, y, z, t)$ are made from combinations of quark and gluon fields which transform according to an irreducible representation (irrep) of the symmetry group, and the angular brackets denote averaging over the correct thermal distribution of fields. We shall also have occasion to use the meson susceptibilities

$$
\chi=\sum_{z} C(z)=\left\langle\frac{N_{z}}{V} \sum_{x, y, z, t} M(0) M^{\dagger}(x, y, z, t)\right\rangle .
$$

Detailed discussions of the isometries of the lattice and irreps of the transfer matrix can be found for gluon operators in 16, 17 and staggered quark operators in [15]. Here we only mention the features used in this work.

For operators made entirely out of gluon fields, "glueball" operators in the usual shorthand, the relevant symmetry is that of a slice of the lattice. For screening correlators such a slice includes two spatial directions and the Euclidean time direction. Since rotations of the spatial directions into Euclidean time are disallowed, the full cubic group, $O_{h}$, breaks down to the dihedral group, $D_{4}^{h}$. For bilinear operators constructed from staggered quark fields, the "meson" operators, the symmetries of the transfer matrix are more complicated due to mixing of spin and flavor components 


\begin{tabular}{|c|c|c|c|c|c|}
\hline$G R F$ & $\check{G}$ & $D_{4}^{h}$ & Operator & $\operatorname{spin} \otimes$ flavor & Particle \\
\hline $\mathbf{1}^{++}$ & $\mathbf{1}_{0}$ & $A_{1}^{+}$ & $\bar{\chi}(r) \chi(r)$ & $1 \otimes 1$ & $\pi, f_{0}(\sigma)$ \\
\hline $\mathbf{1}^{+-}$ & $\mathbf{1}_{0}$ & $A_{1}^{+}$ & $\eta_{z}(r) \zeta_{z}(r) \bar{\chi}(r) \chi(r)$ & $\gamma_{z} \gamma_{5} \otimes \gamma_{z} \gamma_{5}, \gamma_{5} \otimes \gamma_{5}$ & $\pi$ \\
\hline \multirow[t]{3}{*}{$3^{\prime \prime \prime \prime+-}$} & $\mathbf{1}_{4}$ & $A_{1}^{+}$ & $\epsilon(r) \eta_{t}(r) \zeta_{t}(r) \bar{\chi}(r) \chi(r)$ & $\gamma_{i} \gamma_{5} \otimes \gamma_{i} \gamma_{5}, \gamma_{i} \gamma_{z} \otimes \gamma_{i} \gamma_{z}$ & $\rho, a_{1}$ \\
\hline & $\mathbf{2}_{4}$ & $A_{1}^{+}$ & $\epsilon(r)\left[\eta_{x}(r) \zeta_{x}(r)+\eta_{y}(r) \zeta_{y}(r)\right] \bar{\chi}(r) \chi(r)$ & & \\
\hline & & $B_{1}^{+}$ & $\epsilon(r)\left[\eta_{x}(r) \zeta_{x}(r)-\eta_{y}(r) \zeta_{y}(r)\right] \bar{\chi}(r) \chi(r)$ & & \\
\hline \multirow[t]{3}{*}{$3^{\prime \prime \prime \prime++}$} & $\mathbf{1}_{4}$ & $A_{1}^{+}$ & $\epsilon(r) \eta_{z}(r) \zeta_{z}(r) \eta_{t}(r) \zeta_{t}(r) \bar{\chi}(r) \chi(r)$ & $\gamma_{i} \gamma_{k} \otimes \gamma_{j} \gamma_{k}, \gamma_{i} \otimes \gamma_{i}$ & $\rho, b_{1}$ \\
\hline & $\mathbf{2}_{4}$ & $A_{1}^{+}$ & $\epsilon(r) \eta_{z}(r) \zeta_{z}(r)\left[\eta_{x}(r) \zeta_{x}(r)+\eta_{y}(r) \zeta_{y}(r)\right] \bar{\chi}(r) \chi(r)$ & & \\
\hline & & $B_{1}^{+}$ & $\epsilon(r) \eta_{t}(r) \zeta_{t}(r)\left[\eta_{x}(r) \zeta_{x}(r)-\eta_{y}(r) \zeta_{y}(r)\right] \bar{\chi}(r) \chi(r)$ & & \\
\hline \multirow[t]{2}{*}{$3^{-+}$} & $\mathbf{1}_{6}$ & $A_{2}^{-}$ & $\eta_{t}(r) \bar{\chi}(r) D_{t} \chi(r)$ & $\gamma_{i} \otimes 1, \gamma_{i} \gamma_{k} \otimes \gamma_{z} \gamma_{5}$ & $\omega, b_{1}$ \\
\hline & $\mathbf{2}_{2}$ & $E^{-}$ & $\eta_{x, y}(r) \bar{\chi}(r) D_{x, y} \chi(r)$ & & \\
\hline \multirow[t]{2}{*}{$3^{--}$} & $\mathbf{1}_{6}$ & $A_{2}^{-}$ & $\eta_{z}(r) \zeta_{z}(r) \eta_{t}(r) \bar{\chi}(r) D_{t} \chi(r)$ & $\gamma_{i} \gamma_{z} \otimes \gamma_{z}, \gamma_{i} \gamma_{5} \otimes \gamma_{5}$ & $\rho, a_{1}$ \\
\hline & $\mathbf{2}_{2}$ & $E^{-}$ & $\eta_{z}(r) \zeta_{z}(r) \eta_{x, y}(r) \bar{\chi}(r) D_{x, y} \chi(r)$ & & \\
\hline \multirow[t]{2}{*}{$3^{\prime \prime--}$} & $\mathbf{1}_{2}$ & $A_{2}^{-}$ & $\epsilon(r) \zeta_{t}(r) \bar{\chi}(r) D_{t} \chi(r)$ & $\gamma_{5} \otimes \gamma_{i} \gamma_{5}, \gamma_{z} \otimes \gamma_{i} \gamma_{z}$ & $\pi$ \\
\hline & $\mathbf{2}_{0}$ & $E^{-}$ & $\epsilon(r) \zeta_{x, y}(r) \bar{\chi}(r) D_{x, y} \chi(r)$ & & \\
\hline \multirow[t]{2}{*}{$3^{\prime \prime-+}$} & $\mathbf{1}_{2}$ & $A_{2}^{-}$ & $\eta_{z}(r) \zeta_{z}(r) \epsilon(r) \zeta_{t}(r) \bar{\chi}(r) D_{t} \chi(r)$ & $\gamma_{z} \gamma_{5} \otimes \gamma_{j} \gamma_{k}, 1 \otimes \gamma_{i}$ & $\pi, a_{0}$ \\
\hline & $\mathbf{2}_{0}$ & $E^{-}$ & $\eta_{z}(r) \zeta_{z}(r) \epsilon(r) \zeta_{x, y}(r) \bar{\chi}(r) D_{x, y} \chi(r)$ & & \\
\hline
\end{tabular}

TABLE III: Representations of local and one-link separated staggered mesons that were used in this study. The table is extracted from 15. Here $D_{\mu} \phi(\mathbf{x})=\phi(\mathbf{x}+\hat{\mu})+\phi(\mathbf{x}-\hat{\mu})$. We have chosen $\eta_{x}=(-1)^{y+z+t}, \eta_{y}=(-1)^{z+t}, \eta_{z}=(-1)^{t}, \eta_{t}=1$, and $\zeta_{x}=1, \zeta_{y}=(-1)^{x}, \zeta_{z}=(-1)^{x+y}, \zeta_{t}=(-1)^{x+y+z}$. The spin/flavor and particle assignments at $T=0$ are standard. $G R F$ is the symmetry group of the $T=0$ staggered fermion transfer matrix and $\breve{G}$ that at $T>0$.

and staggering of the quark fields. However, this transfer matrix also carries representations of $D_{4}^{h}$ as shown in [15]. $D_{4}^{h}$ has eight one-dimensional and two two-dimensional irreps.

In the continuum, the $T=0$ symmetry group is that of $O(3)$ rotations of the slice, and breaks down for $T>0$ to the symmetry group of the cylinder, $\mathcal{C}=O(2) \times Z_{2}$. The lattice groups are subgroups of these continuum groups. The real irreps of $\mathcal{C}$ are easily obtained. There are two one-dimensional irreps $0_{+}$and $0_{-}$, which come from the $J_{z}=0$ components of the even and odd spin representations of $O(3)$. There is a countable infinity of two-dimensional real irreps which span the spaces of $J_{z}= \pm M$ for any $J>0$. These also carry irreps of the remaining $Z_{2}$ subgroup which corresponds to reflections, $t \rightarrow-t$, in Euclidean time.

In the continuum limit the scalar irrep $A_{1}^{+}$and a non-trivial irrep $A_{2}^{-}$of $D_{4}^{h}$ both collapse into the $0_{+}$irrep of the cylinder group; hence these screening masses must be degenerate in this limit. The $0_{-}$gets both the $A_{1}^{-}$and $A_{2}^{+}$, and hence this pair of screening masses must also become degenerate. The four remaining one-dimensional irreps $B_{1,2}^{ \pm}$ collapse into the $J_{z}= \pm 2$ irrep of $O(2)$, and the two-dimensional irreps $E^{ \pm}$become the $J_{z}= \pm 1$ of $O(2)$. All these patterns of degeneracies have been verified in the glue sector of the $S U(2)$ pure gauge theory [17].

These seemingly esoteric group theoretic constructions actually have very simple physical applications. It has long been appreciated that in screening phenomena, particles of different spin may mix, and that different polarization components may have different dispersion relations [18]. These correspond, respectively, to mixing of equal $J_{z}$ for different $J$ and the distinction between different $J_{z}$ for the same $J$. While we discuss only physics for $T>0$ and zero chemical potential here, it should be noted that the essential group theory lies in the inequivalence of the spatial and temporal directions. This is also true at finite chemical potential, and hence this group theory is also relevant to such future lattice computations.

\section{B. Results}

We have investigated the usual "local" staggered meson operators, i.e., those in which the quark and the anti-quark both land on the same lattice site (see Table IIII). These are familiar from their $T=0$ characters. The scalar $\mathbf{1}^{++}$, $\mathrm{S}$, and the pseudo-scalar $\mathbf{1}^{+-}$, PS, both belong to the $A_{1}^{+}$of $D_{4}^{h}$. The three components of the vector $\mathbf{3}^{\prime \prime \prime \prime}{ }^{++}, \mathrm{V}$, and the axial-vector $3^{\prime \prime \prime \prime}{ }^{+-}, \mathrm{AV}$, can be further reduced. These give two different copies of $A_{1}^{+}$, and also a $B_{1}^{+}$. All the $A_{1}^{+}$irreps have been measured extensively before.

We have also made the first ever $T>0$ investigation of a class of "non-local" staggered mesons, one in which the quark and the anti-quark are separated by one link (see Table III). These are made gauge invariant by including any 
product of links that starts on one of these sites and ends on another. The various operators lie in the $A_{2}^{-}$and $E^{-}$ irreps of $D_{4}^{h}$.

\begin{tabular}{|c||c||c|c|c||c|c|c|}
\hline \multicolumn{1}{|c||}{ Irrep } & $T / T_{c}$ & \multicolumn{3}{c||}{$\mathrm{S} / \mathrm{PS}$} & \multicolumn{3}{c|}{$\mathrm{V} / \mathrm{AV}$} \\
\hline & & $N_{f}=0$ & $N_{f}=2$ & $N_{f}=4$ & $N_{f}=0$ & $N_{f}=2$ & $N_{f}=4$ \\
\hline \multirow{2}{*}{$A_{1}^{+}$} & 1.5 & $4.21 \pm 0.01$ & $3.67 \pm 0.02$ & $3.51 \pm 0.01$ & $5.32 \pm 0.08$ & $5.44 \pm 0.08$ & $5.6 \pm 0.1$ \\
& 2.0 & $4.528 \pm 0.008$ & $4.08 \pm 0.01$ & $3.744 \pm 0.001$ & $5.64 \pm 0.02$ & $5.72 \pm 0.04$ & $5.6 \pm 0.1$ \\
& 3.0 & $4.84 \pm 0.04$ & $4.340 \pm 0.008$ & $4.29 \pm 0.01$ & $5.656 \pm 0.008$ & $5.72 \pm 0.03$ & $5.6 \pm 0.1$ \\
\hline \multirow{2}{*}{$A_{2}^{-}$} & 1.5 & $4.6 \pm 1.1$ & $4.6 \pm 1.0$ & $\times$ & $4.4 \pm 1.1$ & $4.4 \pm 1.0$ & $\times$ \\
& 2.0 & $4.4 \pm 0.8$ & $4.1 \pm 0.8$ & $4.1 \pm 1.2$ & $4.4 \pm 1.0$ & $3.6 \pm 1.2$ & $3.9 \pm 0.7$ \\
& 3.0 & $4.4 \pm 0.6$ & $4.8 \pm 0.8$ & $5.2 \pm 2.0$ & $5.0 \pm 0.6$ & - & $5.6 \pm 2.8$ \\
\hline
\end{tabular}

TABLE IV: Screening masses in units of temperature, $M / T$, in various "meson" channels. A cross indicates that no data exists, and a dash that no stable measurement was possible. The $N_{f}=4$ results are from runs reported in [12].

Chiral symmetry restoration gives the following relations between the staggered local meson correlators-

$$
C_{P S}(z)=(-1)^{z} C_{S}(z) \quad \text { and } \quad C_{A V}(z)=(-1)^{z} C_{V}(z) .
$$

We found that these relations are satisfied for almost all the correlators to great accuracy at all $T$. The only small discrepancy is for the S/PS correlators at $T=1.5 T_{c}$, where the relation is violated at the $68 \%$ confidence level (but not at the $95 \%$ confidence level). In order to rule out the finite MD-time step errors as the source of this oddity, we ran a second simulation at this coupling with half the time step. The results remained unchanged. Since our error estimates are stable, we performed another run with $N_{z}=24$. On this longer lattice the S and PS agreed at the $68 \%$ confidence level.

For staggered Fermions, correlators are usually fitted to a form such as

$$
C(z)=A_{1} \cosh \left[M_{1}\left(z-N_{z} / 2\right)\right]+(-1)^{z} A_{2} \cosh \left[M_{2}\left(z-N_{z} / 2\right)\right] .
$$

The correlator identities in (3) imply that the combinations $C_{V}+C_{A V}$ and $C_{V}+(-1)^{z} C_{A V}$ project out the mass eigenstates in the chiral symmetric phase. We have also made single mass fits to these projections to check for stability of the fitted parameters. In addition we have extracted screening masses by constructing local masses with these projections. The local mass, $m(z)$, is defined by the solution of

$$
\frac{\cosh \left[m(z)\left(z-1-N_{z} / 2\right)\right]}{\cosh \left[m(z)\left(z+1-N_{z} / 2\right)\right]}=\frac{C(z-1)}{C(z+1)},
$$

where the correlation functions are estimated by a jack-knife procedure. In order to take care of covariance between various measurements, we take the number of jack-knife bins to be much smaller than the number of measurements.

In the $N_{f}=4$ theory the $B_{1}$ correlators are identically zero 11. A statistical test of this hypothesis is made through the usual

$$
\chi^{2}=\sum_{i j}\left(y_{i}-h_{i}\right)\left(\sigma^{-1}\right)_{i j}\left(y_{j}-h_{j}\right),
$$

where $y_{i}$ are the measurements of the correlators at different $z, \sigma$ is the covariance of these measurements, and the hypothesis being tested is that $h_{i}=0$. With the data we have gathered in $N_{f}=2 \mathrm{QCD}$, we found that

$$
\chi^{2}= \begin{cases}15.8 & \left(1.5 T_{c}\right) \\ 13.2 & \left(2.0 T_{c}\right) \\ 24.1 & \left(3.0 T_{c}\right)\end{cases}
$$

Since $N_{z}=16$ in all these measurements, the number of degrees of freedom is 15 . Thus, no alternative hypothesis can be supported by our data at the $99 \%$ confidence limit for $T \leq 2 T_{c}$. For $T=3 T_{c}$, where there might be a signal, a one-mass fit by the first term of eq. (1) gave a coefficient about $3 \sigma$ away from zero, and a screening mass $M / T=4.5 \pm 0.2$. In the quenched theory, on the other hand, the situation was more like the $N_{f}=4$ theory. At all $T$ the $B_{1}^{+}$correlator was compatible with zero. The fits also gave similar agreement with a vanishing correlator. More detailed studies of the $B_{1}$ correlator with high statistics at several temperatures above $2 T_{c}$ might be of interest in the future. 
The correlation functions in the remaining irreps are non-trivial. The screening masses obtained are listed in Table IV], where we have also collected measurements made with quenched and $N_{f}=4$ dynamical staggered Fermions on $N_{t}=4$ lattices. Note that the two sets of $A_{1}^{+}$correlators, the S/PS and the $\mathrm{V} / \mathrm{AV}$, give different screening masses. The V/AV screening mass, $M_{V}$, is consistent with free field theory. At all $T$, the $A_{2}^{-}$screening mass is close to the $\mathrm{S} / \mathrm{PS}$ screening mass, $M_{S}$, but due to the large errors, is also consistent with $M_{V}$. The $E^{-}$screening mass is harder to determine because the correlator is very noisy; the central value is consistent with free field theory, but has very large errors.

The two parity projected local V and AV correlators are identical. This implies that not only the screening masses, but also the mixture of states excited by the operator are identical. In other words, the $a_{1}$ and $b_{1}$ screening states mix for $T>T_{c}$. Similar equalities were found in the parity projections of the $A_{2}^{-}$(and $E^{-}$) correlators coming from the $\mathrm{V}$ and AV non-local meson operators, showing that the $\rho$ and $\omega$ screening states also mix. The non-local $\mathrm{S}$ and PS correlators similarly bear evidence for the mixing of $\pi$ and $a_{0}$. In addition, the equality of the $A_{2}^{-}$screening mass determined from all four $A_{2}^{-}$correlators at our disposal also argues for a mixing of $\pi$ and the $J_{z}=0$ component of the $\rho / \omega$. Thus both the phenomena expected in screening correlators are seen - mixing of states of different $J$ and different components of the same $J$ having different dispersion relations.

\section{QUARK NUMBER SUSCEPTIBILITIES}

\section{A. Definitions}

We define the partition function

$$
Z=\int \mathcal{D} U \mathrm{e}^{-S_{g}} \operatorname{det} M\left(m_{u}, \mu_{u}\right) \operatorname{det} M\left(m_{d}, \mu_{d}\right) \operatorname{det} M\left(m_{s}, \mu_{s}\right),
$$

where $S_{g}$ is the gluon action of interest and $M$ denotes appropriate lattice Dirac operators. The chemical potentials for each flavor can be combined into the singlet, triplet and octet $S U(3)$ chemical potentials-

$$
\mu_{0}=\mu_{u}+\mu_{d}+\mu_{s}, \quad \mu_{3}=\mu_{u}-\mu_{d}, \quad \text { and } \quad \mu_{8}=\mu_{u}+\mu_{d}-2 \mu_{s} .
$$

Our notational convention is that indices such as $f$ and $f^{\prime}$ run over the flavors $u, d$ and $s$, and indices such as $i$ and $j$ run over the $S U(3)$ diagonal generators 0,3 and 8 .

The quark number densities are

$$
n_{f} \equiv\left(\frac{T}{V_{3}}\right) \frac{\partial \ln Z}{\partial \mu_{f}}=\left(\frac{T}{V_{3}}\right)\left\langle\operatorname{tr} M_{f}^{-1} M_{f}^{\prime}\right\rangle,
$$

where $M_{f}^{\prime}=\partial M_{f} / \partial \mu_{f}, V_{3}=N_{x}^{3} a^{3}$, and $T=1 / N_{t} a$. Conversion to the other basis simply follows using the definitions in eq. (9) and the chain rule of differentiation. Note that $n_{0}=\left(n_{u}+n_{d}+n_{s}\right) / 3$ and $n_{3}=\left(n_{u}-n_{d}\right) / 2$ are the baryon number and isospin densities, respectively. These are densities of (conserved) charges and not of quark number. The quark number susceptibilities are the second derivative of the free energy with respect to the chemical potentials

$$
\chi_{f f^{\prime}} \equiv \frac{\partial n_{f}}{\partial \mu_{f^{\prime}}}=\left(\frac{T}{V_{3}}\right)\left[\frac{1}{Z} \frac{\partial^{2} Z}{\partial \mu_{f} \partial \mu_{f^{\prime}}}-\frac{1}{Z} \frac{\partial Z}{\partial \mu_{f}} \frac{1}{Z} \frac{\partial Z}{\partial \mu_{f^{\prime}}}\right] .
$$

To lighten the notation, we shall put only one subscript on the diagonal parts of $\chi$.

We are interested in evaluating the susceptibilities for $m_{u}=m_{d}<m_{s}$ at the point $\mu_{f}=0$ for all $f$, yielding much simplification. For example, each $n_{f}$ vanishes, a fact that we utilize as a check on our numerical evaluation. Moreover, the product of the single derivative terms in eq. (11) vanishes, since each is proportional to a number density. Finally, since the masses are degenerate, $M\left(m_{u}, 0\right)=M\left(m_{d}, 0\right)$ for each configuration of gauge links under study.

Flavor off-diagonal susceptibilities such as

$$
\chi_{u d}=\left(\frac{T}{V_{3}}\right)\left\langle\operatorname{tr} M_{u}^{-1} M_{u}^{\prime} \operatorname{tr} M_{d}^{-1} M_{d}^{\prime}\right\rangle
$$

are given entirely in terms of the expectation values of disconnected loops. Such quantities are numerically hard to compute. We discuss their evaluation later. Since $M_{u}=M_{d}$, we obtain $\chi_{u s}=\chi_{d s}$ with each defined by an obvious generalization of the formula above. Of the flavor diagonal susceptibilities we shall use

$$
\chi_{s}=\left(\frac{T}{V_{3}}\right)\left[\left\langle\left(\operatorname{tr} M_{s}^{-1} M_{s}^{\prime}\right)^{2}\right\rangle+\left\langle\operatorname{tr}\left(M_{s}^{-1} M_{s}^{\prime \prime}-M_{s}^{-1} M_{s}^{\prime} M_{s}^{-1} M_{s}^{\prime}\right)\right\rangle\right] .
$$


$\chi_{u}=\chi_{d}$ are given by an obvious generalization of this formula. Numerically, the simplest quantity to evaluate is the diagonal iso-vector susceptibility

$$
\chi_{3}=\frac{1}{2}\left(\frac{T}{V_{3}}\right)\left\langle\operatorname{tr}\left(M_{u}^{-1} M_{u}^{\prime \prime}-M_{u}^{-1} M_{u}^{\prime} M_{u}^{-1} M_{u}^{\prime}\right)\right\rangle .
$$

Two more susceptibilities are of interest. These are the baryon number and electric charge susceptibilities,

$$
\chi_{0}=\frac{1}{9}\left(4 \chi_{3}+\chi_{s}+4 \chi_{u d}+4 \chi_{u s}\right) \quad \text { and } \quad \chi_{q}=\frac{1}{9}\left(10 \chi_{3}+\chi_{s}+\chi_{u d}-2 \chi_{u s}\right) .
$$

Note that $\chi_{0}$ is the baryon number susceptibility for three flavors of quarks. As a result, this expression differs from the iso-singlet quark number susceptibility for two flavors, defined in [6], both in overall normalization and by terms containing strangeness.

Note that quark masses appear in two places: first in the determinant in (8) which defines the weights for the averaging, and second in the trace of the Dirac operators which define the susceptibilities. The first is the dynamical sea quark mass $m$, and the second is the valence quark mass $m_{v}$. In principle these can be different. We work with light sea quark masses for the $u$ and d flavors, as described earlier, and use the quenched approximation for the strange quark, i.e., set $\operatorname{det} M_{s}=1$. We have earlier reported measurements of these susceptibilities when all the flavors are quenched [8]. For staggered quarks each trace in eqs. 12 14 comes with a factor of $1 / 4$ to compensate for flavor doubling. We differ from the conventions of [6] by this overall factor.

These susceptibilities are easy to compute in free field theory i.e., for an ideal Fermi gas. As expected, $n_{f}=0$ for $\mu_{f}=0$. All the off-diagonal susceptibilities are also zero. For $\chi_{3}$ we obtain

$$
\chi_{F F T}=\frac{1}{4 N_{t} N_{x}^{3}} \sum_{p} \frac{\sin ^{2} p_{0} \cos ^{2} p_{0}}{\left[m^{2}+\sum_{\nu} \sin ^{2} p_{\nu}\right]^{2}},
$$

where the spectrum of momenta is $p_{0}=\left(2 \pi / N_{t}\right)\left(n_{0}+1 / 2\right)$, for $n_{0}=0, \cdots, N_{t}-1$, and $p_{\nu}=\left(2 \pi / N_{x}\right) n_{\nu}$, where $n_{\nu}=0, \cdots, N_{x}-1$ for $\nu \neq 0 . \chi_{s}$ is also given by the same expression for the appropriate quark mass. $\chi_{0}^{F F T}$ and $\chi_{q}^{F F T}$ can then be obtained using eq. (15).

\section{B. Optimizing the measurements}

The traces required in measurements of the quark number susceptibility were estimated using a well-known stochastic method. Such stochastic estimators for the traces of $N \times N$ matrices can be constructed from ensembles of $N$ dimensional complex vectors, $R$, of which each component, $z_{\alpha}$, is drawn from a Gaussian distribution of unit variance. From the identity

$$
\frac{1}{2} \int z_{\alpha}^{*} z_{\beta} \prod_{\mu=1}^{N} \mathrm{e}^{-\left|z_{\mu}\right|^{2} / 2}\left(\frac{d^{2} z_{\alpha}}{2 \pi}\right)=\delta_{\alpha \beta}
$$

(here $d^{2} z$ means $r d r d \theta$ and the complex number $z=r \exp i \theta$ ) we construct the estimator by multiplying both sides by a matrix element $A_{\alpha \beta}$ and summing over the indices. The right hand side gives $\operatorname{tr} A$. The integral on the left hand side has an obvious Monte Carlo estimator, giving the relation

$$
\operatorname{tr} A=\frac{1}{2 N_{v}} \sum_{i=1}^{N_{v}} R_{i}^{\dagger} A R_{i}=\overline{R^{\dagger} A R}
$$

where $N_{v}$ is the number of vectors used in making the estimate. Note that eq. (17) implies that the factor 2 above is part of the weight in the average over the ensemble of random complex vectors.

The stochastic estimator for $(\operatorname{tr} A)^{2}$ is a small modification of that given in [6]. We draw $L$ sets of independent random Gaussian vectors as before and construct the estimator

$$
(\operatorname{tr} A)^{2}=\frac{2}{L(L-1)} \sum_{i>j=1}^{L}\left(\overline{R_{i}^{\dagger} A R_{i}}\right)\left(\overline{R_{j}^{\dagger} A R_{j}}\right) .
$$

If we draw $N_{v}$ vectors in the evaluation of the single trace, then it is simplest to divide these into $L$ sets of $N_{v} / L$ vectors for the estimate of each ensemble average in the sum above [23]. 


\begin{tabular}{|c||c|c|c|c||c|c|c|}
\hline \multicolumn{1}{|c||}{$m_{v} / T_{c}$} & \multicolumn{9}{|c||}{$4 \times 8^{3}$} & \multicolumn{3}{|c|}{$4 \times 16^{3}$} \\
\hline & $N_{v}$ & $\chi_{3} / T^{2}$ & $10^{4} \chi_{u d} / T^{2}$ & $\chi_{\pi} / T^{2}$ & $\chi_{3} / T^{2}$ & $10^{4} \chi_{u d} / T^{2}$ & $\chi_{\pi} / T^{2}$ \\
\hline 0.1 & 20 & $0.96(4)$ & $-2(2)$ & $104(6)$ & & & \\
& 40 & $0.96(3)$ & $-2.1(6)$ & & $0.98(3)$ & $0.02(2)$ & $106(6)$ \\
& 100 & $0.96(2)$ & $0.06(4)$ & & & & \\
0.3 & 100 & $0.91(1)$ & $0.06(4)$ & $96(4)$ & $0.91(2)$ & $0.02(2)$ & $96(3)$ \\
0.5 & & $0.84(1)$ & $0.05(3)$ & $88(3)$ & $0.83(1)$ & $0.02(1)$ & $87(2)$ \\
0.75 & & $0.748(9)$ & $0.05(3)$ & $78(2)$ & $0.726(8)$ & $0.02(1)$ & $78(2)$ \\
1.0 & & $0.660(7)$ & $0.04(3)$ & $70(2)$ & $0.639(6)$ & $0.014(8)$ & $70(2)$ \\
\hline
\end{tabular}

TABLE V: Susceptibilities for $T=1.5 T_{c}$ and $m / T_{c}=0.1$. On the larger lattice we used $N_{v}=40 . \chi_{\pi}$ is measured with a single point source by integrating the local PS correlator.

In [6] the sum above was taken over all pairs, and the diagonal term was removed by subtracting a term equal to a stochastic estimator for $\operatorname{tr}\left(A^{2}\right)$. In [8] where that method was used, we showed that reasonably large values, $N_{v} \approx 100$ were necessary to avoid a systematic bias in $\chi_{0}-\chi_{3}$. However, the estimate is facilitated by excluding the diagonal term from the sum above. For example, we found that the error in $\operatorname{tr} A$ scales as $1 / \sqrt{N_{v}}$, whereas that in $(\operatorname{tr} A)^{2} \operatorname{scales}$ as $1 / N_{v}$, for fixed $L$. The dependence on $L$ was marginal, as long $N_{v} / L>5$.

\begin{tabular}{|c||c|c|c|c|c||c|c|c|c|c|}
\hline$m_{v} / T_{c}$ & $T / T_{c}$ & $\chi_{F F T} / T^{2}$ & $\chi_{3} / T^{2}$ & $10^{6} \chi_{u d} / T^{2}$ & $\chi_{\pi} / T^{2}$ & $T / T_{c}$ & $\chi_{F F T} / T^{2}$ & $\chi_{3} / T^{2}$ & $10^{6} \chi_{u d} / T^{2}$ & $\chi_{\pi} / T^{2}$ \\
\hline 0.1 & 1.25 & 1.1274 & $0.90(3)$ & $0(1)$ & $149(10)$ & 2.00 & 1.1276 & $0.973(7)$ & $0(1)$ & $84(2)$ \\
0.3 & & 1.1250 & $0.80(1)$ & $0.4(8)$ & $119(6)$ & & 1.1227 & $0.951(7)$ & $0(1)$ & $82(2)$ \\
0.5 & & 1.1203 & $0.704(8)$ & $0.2(7)$ & $99(4)$ & & 1.1248 & $0.888(9)$ & $1(1)$ & $79(2)$ \\
0.75 & & 1.1112 & $0.598(6)$ & $0.0(6)$ & $83(2)$ & & 1.1212 & $0.849(6)$ & $0.3(8)$ & $75(1)$ \\
1.0 & & 1.0988 & $0.511(6)$ & $0.1(5)$ & $72(2)$ & & 1.1162 & $0.783(6)$ & $0.4(7)$ & $70(1)$ \\
\hline 0.1 & 1.50 & 1.1275 & $0.96(1)$ & $4(3)$ & $99(4)$ & 3.00 & 1.1277 & $0.995(4)$ & $0.7(7)$ & $75(1)$ \\
0.3 & & 1.1259 & $0.903(7)$ & $3(2)$ & $93(3)$ & & 1.1273 & $0.988(4)$ & $0.7(7)$ & $75(1)$ \\
0.5 & & 1.1226 & $0.829(6)$ & $3(2)$ & $85(3)$ & & 1.1264 & $0.973(4)$ & $0.7(7)$ & $74(1)$ \\
0.75 & & 1.1162 & $0.733(4)$ & $2(1)$ & $76(2)$ & & 1.1248 & $0.946(4)$ & $0.6(6)$ & $72(1)$ \\
1.0 & & 1.1074 & $0.646(4)$ & $1.8(9)$ & $69(1)$ & & 1.1226 & $0.912(4)$ & $0.6(6)$ & $70(1)$ \\
\hline
\end{tabular}

TABLE VI: Susceptibilities measured on $4 \times 12^{3}$ lattices for $N_{f}=2$ with $m / T_{c}=0.1$ and $N_{v}=100$. $\chi_{\pi}$ is measured with a single point source by integrating the local PS correlator.

\begin{tabular}{|c|c|c|c|c|}
\hline$m_{v} / T_{c}$ & $\chi_{F F T} / T^{2}$ & $\chi_{3} / T^{2}$ & $10^{6} \chi_{u d} / T^{2}$ & $\chi_{\pi} / T^{2}$ \\
\hline 0.075 & 1.1276 & $0.97(1)$ & $2(2)$ & $106(5)$ \\
0.1 & 1.1275 & $0.97(1)$ & $1(2)$ & $106(4)$ \\
0.3 & 1.1259 & $0.906(8)$ & $1(1)$ & $98(4)$ \\
0.5 & 1.1226 & $0.828(6)$ & $0(1)$ & $90(3)$ \\
0.75 & 1.1162 & $0.729(5)$ & $0.4(8)$ & $80(2)$ \\
1.0 & 1.1074 & $0.641(4)$ & $0.2(6)$ & $72(2)$ \\
\hline
\end{tabular}

TABLE VII: Susceptibilities measured at $T=1.5 T_{c}$ on $4 \times 12^{3}$ lattices for $N_{f}=2$ with $m / T_{c}=0.075$ and $N_{v}=80$.

Since the simulation time increases at least linearly in volume, we need to make a choice of volume which reduces computation time without introducing large artifacts into the measurements. We have made a detailed study of the volume dependence of susceptibility measurements at $T=1.5 T_{c}$. As the data in Table $\mathrm{V}$ clearly show, there is no significant volume dependence in the values of $\chi_{3}$ and $\chi_{u d}$ in going from $4 \times 8^{3}$ to $4 \times 16^{3}$ lattices.

In view of this, we have chosen to make the remaining measurements on $4 \times 12^{3}$ lattices. Close to $T_{c}$ we would expect that the measurements are strongly volume dependent. Based on our estimates of the screening masses we find that even at our smallest temperature of $1.25 T_{c}$ the lattice is more than 10 times larger than the longest correlation 


\begin{tabular}{|c|c|c|c|}
\hline$m_{v} / T_{c}$ & $\chi_{3} / T_{c}^{2}$ & $10^{6} \chi_{u d} / T_{c}^{2}$ & $\chi_{\pi} / T_{c}^{2}$ \\
\hline 0.10 & $0.18(7)$ & $0(1)$ & $462(11)$ \\
0.30 & $0.024(9)$ & $0.1(5)$ & $182(3)$ \\
0.50 & $0.007(4)$ & $0.1(3)$ & $122(2)$ \\
0.75 & $0.002(2)$ & $0.1(2)$ & $89(1)$ \\
1.00 & $0.000(1)$ & $0.0(1)$ & $71.4(8)$ \\
\hline
\end{tabular}

TABLE VIII: Susceptibilities at $T=0$ in units of $T_{c}$, measured on $12^{4}$ lattices for $N_{f}=2$ with $m / T_{c}=0.1$ and $N_{v}=80$

length. This assures us that finite lattice size effects are negligible at the lower end of our temperature range. On the other end of the scale, by the simple expedient of not going beyond $3 T_{c}$, we avoid the finite volume effects such as the onset of spatial deconfinement.

Finally, one must optimize the conjugate gradient stopping criterion, in which the norm of the residual vector is required to be smaller than $\epsilon \sqrt{V}$. We were pleasantly surprised to find that, in thermalized test configurations, results for $\chi_{3}$ and $\chi_{u d}$ changed by less than 1 part in $10^{4}$ on changing $\epsilon$ in the range from 0.01 to $10^{-6}$. Increasing $\epsilon$ by one order of magnitude meant a decrease of CPU time by $20-25 \%$. These numbers were reproduced for test configurations on three lattice volumes, and a variety of $T$. In actual measurements we chose $\epsilon$ to be $10^{-5}$ for $T \geq 1.25 T_{c}$ and $10^{-3}$ otherwise.

\section{Results}

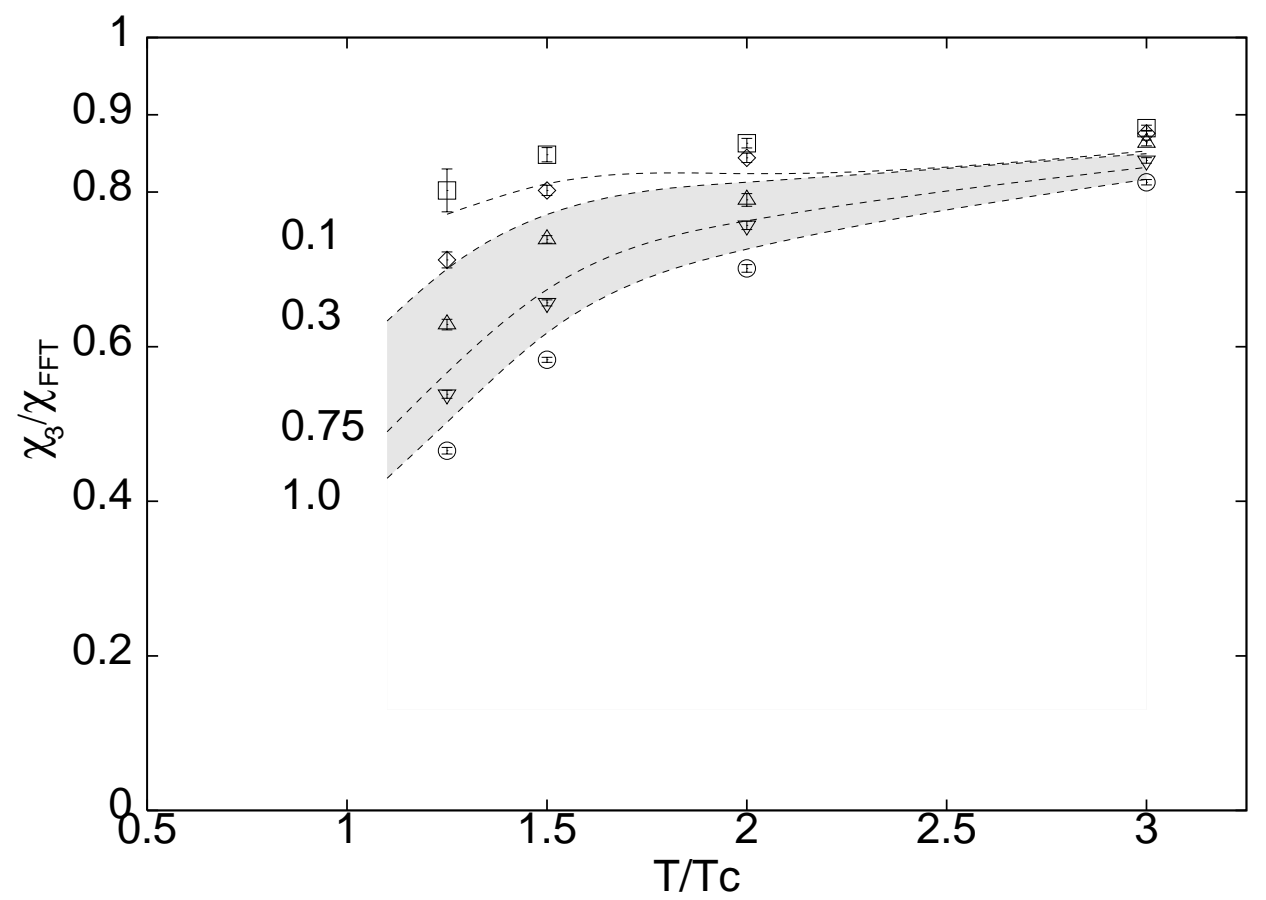

FIG. 2: $\chi_{3} / \chi_{F F T}$ as a function of $T / T_{c}$ for $m_{v} / T_{c}=0.1$ (boxes), 0.3 (diamonds), 0.5 (up triangles), 0.75 (down triangles) and 1 (circles); all for $m / T_{c}=0.1$. For comparison the curves show the measurements in quenched QCD. All the data shown here is taken on $4 \times 12^{3}$ lattices. The shaded area denotes the mass range for strange quarks.

Our main results on the $T$ and $m_{v}$ dependence of $\chi_{3}$ and $\chi_{u d}$ are collected in Table VI for two flavors of dynamical Fermions with $m / T_{c}=0.1$. The results of a simulation at $T=1.5 T_{c}$ with a smaller $m / T_{c}=0.075$ are collected in Table VII. Comparing the two, we see no statistically significant change in $\chi_{3}$ over this range of masses. While $\chi_{3}$ is expected to be strongly dependent on $m$ for $T \simeq T_{c}$ in the chiral limit, our results indicate that the dependence of $\chi$ on the sea quark mass away from the critical region is small. This suggests that the values of $\chi_{3}$ or $\chi_{u d}$ in the chiral 
limit for $T \geq 1.25 T_{c}$ are within errors those in Table VI. Table VIII shows that all the susceptibilities indeed vanish at $T=0$ as expected.

A comparison of the dynamical and quenched theories is displayed in Figure 2 in terms of the ratio $\chi_{3} / \chi_{F F T}$. While the general trend of the data are similar in the two cases, some quantitative differences are visible. The most important of these is in the asymptotic value of $\chi / \chi_{F F T}$. For small $m_{v}$ the ratio is 0.85 at $T / T_{c}=3$ in the quenched theory, whereas it is 0.88 for $N_{f}=2$. For larger $m_{v}, \chi_{3}$ is almost unchanged on unquenching the Fermions. $\chi_{s}$ changes by less than $3 \%$ when the light fermions are unquenched, leading us to believe that the unquenching of the strange quark will not affect this quantity significantly.

These observations are not explained in continuum perturbation theory at high temperature, which yields

$$
\chi / \chi_{F F T}=1-2\left(\frac{\alpha_{S}}{\pi}\right)+8 \sqrt{1+\frac{N_{f}}{6}}\left(\frac{\alpha_{S}}{\pi}\right)^{3 / 2},
$$

when the plasmon term is resummed [19]. Here $\alpha_{S}$ is the strong coupling at a scale appropriate to the temperature $T$. It is easy to see that this ratio is never less than 0.981 for $N_{f}=0\left(0.986\right.$ for $\left.N_{f}=2\right)$. This minimum is reached when $\alpha_{S}=\pi / 6\left(6+N_{f}\right)$. A recent analysis showed that $T_{c} / \Lambda_{\overline{M S}}=1.15 \pm 0.05$ for $N_{f}=0$ and $0.49 \pm 0.02$ for $N_{f}=2[2$. Taking the scale for the strong coupling to be $2 \pi T$, this means that for $N_{f}=2$ the minimum occurs at $T \simeq 3300 \Lambda \frac{}{M S}$ (and $T \simeq 110 \Lambda_{\overline{M S}}$ for $N_{f}=0$ ). Thus in both cases, the weak coupling estimates decrease as a function of temperature in the range studied here, in contrast to our lattice results, which increase. It would be interesting to check whether a full $\mathcal{O}\left(\alpha_{S}^{5 / 2}\right)$ computation for $\chi$ approaches the lattice results, and whether any resummations of the perturbation theory do better [7], 20].

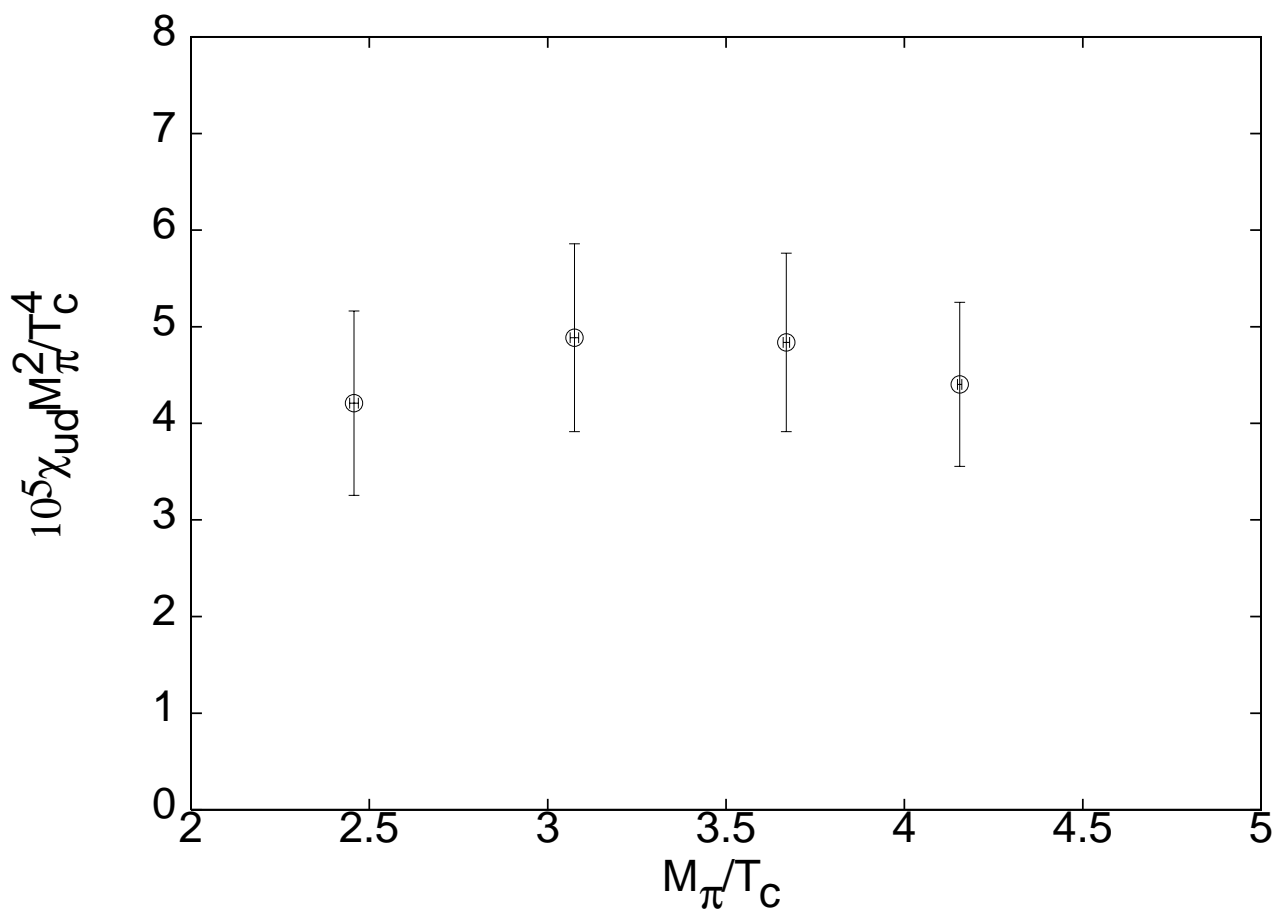

FIG. 3: $\chi_{u d}$ scales as $1 / M_{\pi}^{2}$ for $T=0.75 T_{c}$ in quenched QCD. The measurements of $\chi_{u d}$ and $M_{\pi}$ were both performed on the same configurations on $4 \times 12^{3}$ lattices 8 .

The off-diagonal susceptibility, $\chi_{u d}$, vanishes for $T>T_{c}$ and also at $T=0$. As shown in Table VI, the errors on $\chi_{u d} / T^{2}$ for $T>T_{c}$ are of the order of $10^{-6}$, and hence this is the precision within which this quantity can be said to vanish for all $m_{v}$. Thus, $\chi_{u d}$ and other non-diagonal parts of the flavor space susceptibilities can be totally neglected in constructing other susceptibilities. It is interesting to note that $\chi_{u d}$ is zero in an ideal gas, and perturbative contributions start at order $\alpha_{S}^{2}$. Taking the scale to be $2 \pi T$, as before, and the same values of $T_{c} / \Lambda_{\bar{M} S}$, we might then expect $\chi_{u d}$ to be non-zero at the level of $0.04-0.1$ (for $N_{f}=2$ ) in the temperature range we studied. The substantially smaller, indeed vanishing, value thus accentuates the puzzle about perturbation theory [24].

The only place where $\chi_{u d}$ is definitely non-zero is for $0<T<T_{c}$. A re-analysis of our data in the quenched theory at $T=0.75 T_{c}$ [8] showed that $\chi_{u d}$ differs from zero by over four standard deviations. As displayed in Figure 3 , $\chi_{u d}$ scales inversely with the square of the pion screening mass, $M_{\pi}$, at this temperature. Since it is known that the 
hadron masses are independent of temperature from $T=0$ to some point rather close to $T_{c}$ [21, the only possible temperature dependence of $\chi_{u d}$, for such $T$, would be in the value of the constant $\chi_{u d} M_{\pi}^{2}$. Note that the spatial lattice size is such that $M_{\pi} L \geq 10$, and finite size effects are negligible. In order to ensure this on $4 \times 12^{3}$ lattices, we are constrained to use reasonably heavy quarks. The pion screening mass is then rather large. In future we plan to study $\chi_{u d}$ for $T<T_{c}$ in more detail by pushing towards lower pion mass with larger lattices, covering a bigger range of $T$ and using dynamical quarks.

\section{Relation to screening masses}

The physics of a plasma lies in screening. However, it is not always clear how screening appears in different guises. Interestingly it can be shown that quark number susceptibility is directly related to screening, and therefore the non-perturbative physics in the two are likely to be the same. It is possible to present a deductive argument from first principles and use the data to illustrate it. Instead, we first show how the data prefers a relation between $\chi$ and the S/PS screening mass $M_{S}$, and then give the group theoretical argument that this is not a numerical coincidence.

The variation of $\chi_{3}$ with $m_{v}$ at fixed $T$ is shown in Figure 4 . For large enough $m_{v}$, there is an exponential fall of $\chi_{3}$, which can be qualitatively understood as the effect of Boltzmann factors. It is less obvious that there should be a threshold $m^{*}(T)$ below which $\chi_{3}$ is almost independent of $m_{v}$. The data indicates that $m^{*}(T) \simeq T$. Such a threshold cannot be derived in a weak coupling expansion. Furthermore, this threshold of constancy is exactly what prevents $\chi_{3} / \chi_{F F T}$ from reaching unity as $m_{v} \rightarrow 0$, since Figure 4 is just another representation of the data in Figure 2 .

The only other observed non-perturbative effect in the quark sector of high-temperature QCD is in the screening mass in the S/PS channel, $M_{S}$. That these two effects are related is shown in the second panel of Figure 4 , where we plot $\chi_{3}$ against $M_{S} / T$. A simple exponential relation between them is seen. The threshold we saw before disappears into the relation between $m_{v}$ and $M_{S}$. The remaining question is why the two should be related at all.

The answer is simple, and relies on the group theoretical classification of screening correlators and masses according to the symmetries of the $T>0$ transfer matrix in the spatial direction [15]. First, from eq. (14) it can be seen that $\chi_{3}$ is the thermal expectation value of a product of two quark propagators sandwiching $\gamma_{0} U$ and summed over all distances. As a result, this quantity can be related to a susceptibility constructed from a one-link separated meson screening correlator. In the $T=0$ notation, this turns out to be a component of the one-link $\mathrm{V} \mathbf{3}^{-+}$. For $T>0$ this component reduces to the irrep $A_{2}^{-}$of the appropriate symmetry group $D_{4}^{h}$ (see Table III). Other correlators which lie in the same irrep are the one-link separated S and PS $\mathbf{3}^{\prime \prime- \pm}$ and the a specific component of the one-link AV $\mathbf{3}^{--}$. In the continuum limit the spectrum of $A_{2}^{-}$screening masses is degenerate with that of the $A_{1}^{+}$, since they come from the same irrep of the continuum $O(2)$ symmetry 17]. The smallest $A_{1}^{+}$screening mass comes from the S/PS local propagators which are used to extract $M_{S}$. This, is the reason for the close relation between $\chi_{3}$ and $M_{S}$ shown in Figure 4 .

Further numerical evidence in favor of this group theoretical argument is the similarity in the relation between $\chi_{\pi}\left(M_{S}\right)$ and $\chi_{3}\left(M_{S}\right)$, as shown in Figure 4 . The near equality of the slopes lends support to our earlier observation that the $A_{2}^{-}$screening mass seems to be equal to $M_{S}$. We expect that for smaller lattice spacing or on using a Fermion action which restores the full flavor symmetry, the two screening masses should become equal. In this limit, the lines would be exactly parallel and the difference in values of $\chi_{\pi}$ and $\chi_{3}$ at the same $M_{S}$ would only reflect different operator overlaps with the same state. This, in fact, is a prediction which should be tested in future.

One further piece of information fits neatly into this group theoretic framework. $\chi_{u d}$ has the same symmetry as $\chi_{3}$ and therefore it is also related to the $\mathrm{S} / \mathrm{PS}$ correlators at all non-zero temperatures. Since it vanishes for $T>T_{c}$, the relation is trivial there. However, for non-zero $T<T_{c}$, we have observed that $\chi_{u d}=Z / M_{\pi}^{2}$. This relation would be as mysterious as that between $\chi_{3}$ and $M_{S}$ in the absence of the argument given above.

It is interesting to note that the preceding arguments rest entirely on the group theory of screening correlators and masses, i.e., on the spatial direction transfer matrix of the finite temperature system. In terms of the Euclidean time direction transfer matrix, the group theory is the same as at $T=0$ and there is no reason for the one-link separated vector temporal correlator to be related to the S/PS. Thus, the physics of quark number susceptibility is related to screening correlators and not to temporal correlators.

\section{DISCUSSION AND SUMMARY}

\section{A. Event-to-event fluctuations}

Since $\chi_{q}$ is related to charge fluctuations, it can be observed experimentally in heavy-ion collisions $\llbracket$. The baryon susceptibility, $\chi_{0}$, and the strange quark susceptibility, $\chi_{s}$, can also be used to measure fluctuations of the 

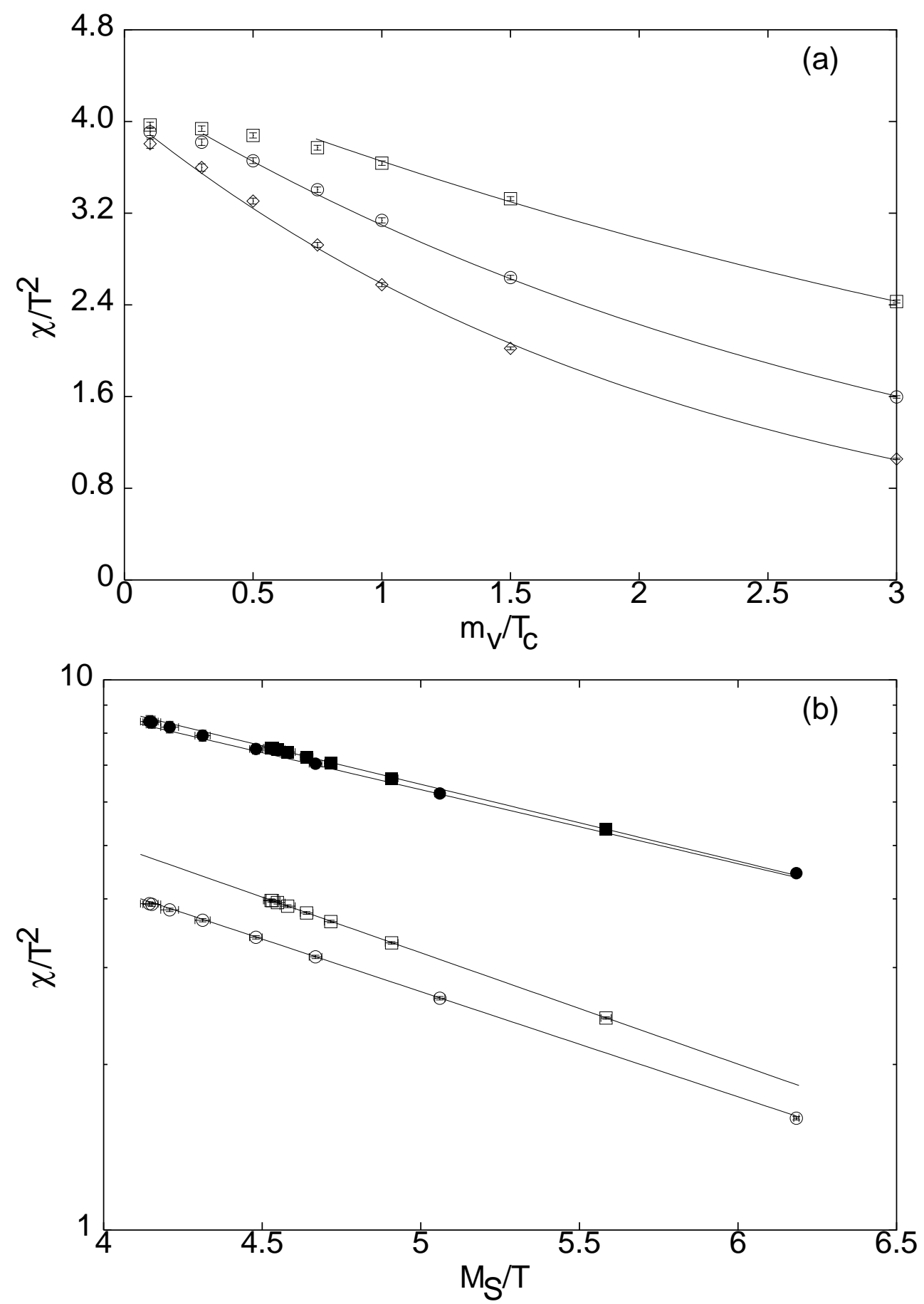

FIG. 4: Panel (a) shows $4 \chi_{3} / T_{c}^{2}$ as a function of $m_{v} / T_{c}$ (for sea quark mass of $0.1 T_{c}$ ) at $T=1.5 T_{c}$ (diamonds), $2 T_{c}$ (circles) and $3 T_{c}$ (boxes). Panel (b) shows $4 \chi_{3} / T^{2}$ (open symbols) and $\chi_{\pi} / 10 T^{2}$ (filled symbols) as a function of $M_{S} / T$ at $2 T_{c}$ (circles) and $3 T_{c}$ (boxes). The lines are exponential fits.

corresponding quantities [5]. Also, $\chi_{s}$ is related by a fluctuation-dissipation theorem to the total amount of strangeness produced in equilibrium, and hence can be used to probe departures from equilibrium in heavy-ion collisions. Given the relevance of these quantities to the experimental search for the quark-gluon plasma, we have collected our results in an easily usable form in Table IX.

The behavior of both $\chi_{q}$ and $\chi_{0}$ above $T_{c}$ are essentially controlled by $\chi_{3}$. It is clear from eq. (15), that for $T \gg T_{c}$, where $\chi_{3} \simeq \chi_{s} \gg \chi_{u d} \simeq \chi_{u s}$, we must have $\chi_{q} / \chi_{0} \approx 2$. Closer to $T_{c}$, but still in the hot phase, if $\chi_{s}$ is also neglected, we would have $\chi_{q} / \chi_{0} \approx 5 / 2$. In fact, we find that this ratio decreases from $2.13 \pm 0.08$ at $1.25 T_{c}$ to $2.02 \pm 0.01$ at $3 T_{c}$. 


\begin{tabular}{|c|c|c|c|c|}
\hline$T / T_{c}$ & $\chi_{3} / \chi_{3}^{F F T}$ & $\chi_{s} / \chi_{s}^{F F T}$ & $\chi_{0} / \chi_{0}^{F F T}$ & $\chi_{q} / \chi_{q}^{F F T}$ \\
\hline 1.25 & $0.80(3)$ & $0.538(5)$ & $0.71(2)$ & $0.76(2)$ \\
1.50 & $0.848(9)$ & $0.657(4)$ & $0.785(6)$ & $0.817(8)$ \\
2.00 & $0.863(6)$ & $0.757(6)$ & $0.828(5)$ & $0.846(5)$ \\
3.00 & $0.883(4)$ & $0.841(4)$ & $0.869(3)$ & $0.877(3)$ \\
\hline
\end{tabular}

TABLE IX: Susceptibilities (in units of their values for an ideal gas) obtained for degenerate dynamical u and d quarks of mass $17 \mathrm{MeV}$ and quenched strange quarks with $m=125 \mathrm{MeV}$. Susceptibilities below $T_{c}$ are discussed in the text.

This shows that $\chi_{s}$ is significant at all temperatures in the plasma, but certainly becomes closer to $\chi_{3}$ with increasing $T$ (Figure 2). In any case, charge fluctuations are twice as large as baryon number fluctuations. Interestingly, we find that $\chi_{q} / \chi_{s}$ also falls from $0.95 \pm 0.03$ at $T=1.25 T_{c}$ to $0.696 \pm 0.004$ at $T=3 T_{c}$ (it is $2 / 3$ in the $T \rightarrow \infty$ limit); therefore strangeness fluctuations become more significant than charge fluctuations with increasing $T$.

In the low-temperature phase, $0<T<T_{c}$, fluctuations are dominated by the flavor off-diagonal contribution. We have displayed evidence (Figure 3) that $\chi_{u d} \simeq 1 / m_{\pi}^{2}$, i.e., fluctuations are due to the lightest particle of given flavor. Although the value of $\chi_{u d}$ is extremely small, the measure of fluctuation is the ratio of $\chi$ and the entropy density [4. While there are no reliable measurements of the entropy for $T<T_{c}$, it is known to be small. It is presently an open question whether the ratio is smaller for $T<T_{c}$ or on the other side of the QCD phase transition.

Assuming that the inverse relation between $\chi_{u d}$ and the pion mass generalizes to other flavor off-diagonal susceptibilities, eq. (15) can be used to predict that $\chi_{q} / \chi_{0} \approx 1 / 4$ for $T<T_{c}$. Corrections to this number are then given in terms of $\left(m_{\pi} / m_{K}\right)^{2}$ and $\left(m_{\pi} / m_{\eta}\right)^{2}$. These might further lower the ratio by $15-20 \%$. As a result, in the low-temperature phase we would obtain the hierarchy of fluctuations - $\chi_{0}>\chi_{q}>\chi_{s}$, in total contrast to the inverted hierarchy $\chi_{0}<\chi_{q}<\chi_{s}$ that we have measured above $T_{c}$.

\section{B. Screening and susceptibilities}

In Euclidean quantum field theories at $T=0$, transfer matrices in all directions are isomorphic, and one, for example, can focus on the time-direction transfer matrix- $\mathbf{T}=\exp (-\mathrm{Ha})$ (here $H$ is the Hamiltonian and $a$ the lattice spacing). The eigenvalues of $\mathbf{T}$ determine the hadron spectrum. The transfer matrix and, hence, its eigenvalues do not change with temperature. Specifically, the symmetry of the transfer matrix remains the rotational $O(3)$ symmetry which is used to classify particle states at $T=0$.

At finite temperature or chemical potential all directions are not equivalent. As a result, $\mathbf{T}$ is not the same as any spatial direction transfer matrix. These have a totally different symmetry, $\mathcal{C}$, that of the cylinder, as discussed extensively in the literature [15, 16, 17]. If the two lowest eigenvalues belonging to the scalar of $\mathcal{C}$ become degenerate, then a phase transition occurs. In general, the eigenvalues of this transfer matrix determine screening masses in equilibrium. Breaking of $O(3)$ to $\mathcal{C}$ implies strange phenomena like the 'mixing' of different spins, or different states of the same spin taking on independent dispersion relations [18]. While these phenomena are strange, they are not new - the group theory is familiar from the textbook examples of the comparison between the hydrogen atom and the hydrogen molecular ion $\mathrm{H}_{2}^{+}$.

Lattice studies of screening correlators have hovered on the verge of this phenomenology. If it has not received widespread attention in the past, that is merely due to the practical difficulties of measuring some of the non-trivial irreps of $\mathcal{C}$, as we show for the first time in Table IV. The equality of parity projected correlators was used in Section IIIB to point out that the $\rho$ and $\omega$ mix in screening, as do the $a_{1}$ and $b_{1}$. The equality of screening masses also gives some evidence for the mixing of $\pi$ with the $J_{z}=0$ component of the $\rho / \omega$, causing a splitting in the screening masses of different components of the latter. We argued in Section IVD that the observed relation between $\chi_{3}$ and the screening mass $M_{S}$ or between $\chi_{u d}$ and $M_{\pi}$ comes from precisely the same physics as the mixing of different spins at finite temperature. The same argument also implies that since fluctuations are observable, screening phenomena are physical.

\section{Future directions}

Several directions for future work are clear, and have been discussed in the body of the paper. Here we collect what seems to us the most important and fruitful possibilities. 
One direction for future work is to examine as many of the non-local screening correlators as possible, in order to gather further information on all the quantum numbers that screening correlators can come in 15 . As we have seen, this requires large lattices and immense statistics, and may well profit from the use of new noise reduction techniques and improved actions which do not change the symmetries (or the positivity) of the transfer matrix.

The ratio $\chi_{3} / \chi_{F F T}$ is not explained in perturbation theory. We noted this in a quenched computation $[8]$, and have verified it in the dynamical QCD computation here. The small difference (roughly 3\%) between the quenched and dynamical results is also significant. Explanation of these results stand as invitations to those who resum the continuum high-temperature perturbation theory. Future lattice computations will need to push towards the continuum limit. Work in this direction is in progress, and will be reported soon.

As explained before, $\chi_{u d}$ vanishes in an ideal gas of quarks, but in an interacting theory would take on a value of order $\alpha_{s}^{3}$. This is several orders of magnitude larger than the largest result that our measurements can tolerate. This observation also is an invitation to perturbation theory.

The off-diagonal susceptibilities are non-vanishing only in the range $0<T<T_{c}$. This region of temperature is hard to study, since all the complications of $T=0$ QCD remain, and none of the simplifications of $T>T_{c}$ QCD start. On the other hand it is important input to studies of fluctuations in heavy ion collisions. We have reported a first quantitative observation here. Future work will push towards more realistic quark masses, taking the thermodynamic limit, and examining a larger range of temperatures. 
[1] A. Ali Khan et al., Phys. Rev. D 63 (2001) 034502; F. Karsch et al., Nucl. Phys. B 605 (2001) 579.

[2] S. Gupta, Phys. Rev. D 64 (2001) 034507.

[3] F. Karsch et al., Phys. Lett. B 478 (2000) 447; A. Ali Khan et al., Phys. Rev. D 64 (2001) 074510.

[4] M. Asakawa et al., Phys. Rev. Lett. 85 (2000) 2072; S. Jeon and V. Koch, ibid. 85 (2000) 2076.

[5] S. Gavin and C. Pruneau, nucl-th/9907040; S. Jeon et al., nucl-th/0105035.

[6] S. Gottlieb et al., Phys. Rev. Lett. 59 (1987) 1513.

[7] J. P. Blaizot et al., Phys. Rev. D 63 (2001) 065003; J. O. Andersen et al., Phys. Rev. D 63 (2001) 105008; K. Kajantie et al., Phys. Rev. Lett. 86 (2001) 10.

[8] R. V. Gavai and S. Gupta, Phys. Rev. D 64 (2001) 074506.

[9] S. Gottlieb et al., Phys. Rev. D 35 (1987) 2531.

[10] M. Fukugita et al., Phys. Rev. D 42 (1990) 2936; S. Aoki et al., Phys. Rev. D 57 (1998) 3910; C. Bernard et al., Phys. Rev. D 45 (1992) 3854; S. Gottlieb et al., Phys. Rev. D 47 (1993) 3619; C. Bernard et al., Phys. Rev. D 54 (1996) 4585; S. Gottlieb et al., Phys. Rev. D 55 (1997) 6852; C. Bernard et al., Phys. Rev. D 61 (2000) 054503.

[11] R. V. Gavai and S. Gupta, Phys. Rev. Lett. 83 (1999) 3784.

[12] R. V. Gavai and S. Gupta, Phys. Rev. Lett. 85 (2000) 2068.

[13] S. Gottlieb et al., Phys. Rev. D 38 (1988) 2245.

[14] D. E. Groom et al., Eur. Phys. Jour., C 15 (2000) 1.

[15] S. Gupta, Phys. Rev. D 60 (1999) 094505.

[16] U. Heller et al., Nucl. Phys. B 417 (1994) 289.

[17] S. Datta and S. Gupta, Nucl. Phys. B 534 (1998) 392, and Phys. Lett. B 471 (2000) 382.

[18] S. A. Chin, Ann. Phys. 108 (1977) 301; A. K. Dutt-Mazumdar et al., Phys. Lett. B 399 (1997) 196; G. Wolf et al., Nucl. Phys. A 640 (1998) 243; K. Saito et al., Phys. Lett. B 433 (1998) 243. O. Teodorescu et al., Phys. Rev. C 61 (2000) 051901.

[19] J. I. Kapusta, "Finite-temperature Field Theory", 1989, Cambridge University Press, Cambridge, UK.

[20] J. P. Blaizot, E. Iancu and A. Rebhan, SACLAY-T01/108, TUW-01-26.

[21] S. Gupta, Phys. Lett. B 288 (1992) 171; G. Boyd et al., ibid. B 349 (1995) 170.

[22] In quenched QCD, where $T_{c} \approx 280 \pm 10 \mathrm{MeV}, m_{s} / T_{c}=0.25-0.60$.

[23] We thank K. Kanaya for a discussion on this estimator.

[24] In [20], it is also shown that $\chi_{u d}$ starts at order $\alpha_{S}^{3}$ with a small coefficient, bringing the resummed prediction closer to the data. 